\title{
Role of 3D Hypofractionated Radiotherapy in Treatment of High Grade Gliomas: A Prospective Study
}

\author{
Talaat E. Elmokadem, Mohamed A. Mazrouh , Said M I. Yousef, Bader A.A bdel-Maksoud * \\ Department of Clinical Oncology and Nuclear Medicine, Faculty of Medicine, Zagazig University, Egypt
}

Copyright $@ 2014$ Horizon Research Publishing All rights reserved.

\begin{abstract}
Purpose: To evaluate the efficacy and safety of hypofractionated radiotherapy in treatment of patients with high grade glioma. Patients and Methods: A total of thirty patients with high grade glioma referred to clinical oncology and nuclear medicine department, Zagazig University Hospitals between July 2012 to January 2014. All patients were treated with three dimensional (3D) conformal hypofractionated radiotherapy at a dose of $45 \mathrm{~Gy} / 15 \mathrm{f}$ treatments over 3 weeks for cases with gross total excision followed by 9 Gy in 3 fractions for cases with subtotal excision and biopsy. Results: The median age was 53 years (range 26-66). The median follow up was 12.23 months (range: 4-22 months). One-year overall survival (OAS) and progression free survival (PFS) rates were $58.9 \%$ and $12 \%$, respectively. In univariate analysis, age $\geq 50$ years, poor performance status [Karnofasky score of $\geq 50-70 \%$ ]. Grade IV histology, subtotal excision and overall response to treatment was all associated with statistically significant reduction in OAS and PFS rates. Multivariate analysis, showed that age $<50$ years and performance status $>70$ were the only independent prognostic factors that were associated with good OAS ( $p=0.010$ and $p=0.015$, respectively), and grade III and overall response were the only prognostic factors associated with good PFS ( $p=0.002$ and $p=0.014$, respectively). There were minimal side effects to radiation therapy, and all patients complete treatment time without interruption. Conclusion: Conformal hypo fractionated radiotherapy is effective and well tolerated for patients with high grade glioma.
\end{abstract}

Keywords $\quad$ Hypofractionation, High Grade
Gliomas,3DCRT

\section{Introduction}

High grade gliomas are the most frequent and invasive brain tumors. They occur in all age groups but predominate in the fifth and sixth decades, and they account for $35-45 \%$ of all brain tumors in adults (1). The overall prognosis remains poor for patients with high grade glioma. (2). The survival after surgery alone is approximately 4 months, indicating the need for more efficient and tolerable treatment alternatives (3). Radiotherapy has been the most effective treatment for these aggressive lesions, but generally RT can only delay disease progression or recurrence (4). Shortening the treatment and controlling the symptoms of these patients are important. Conventional RT schemes require 6-7 weeks of treatment (5). Numerous studies have found that shorter course of RT might be an appropriate option for the patients with high grade gliomas, especially those who have poor prognosis (6). Because of the much worse prognosis associated with advanced age, efforts have been made to reduce the intensity and duration of the treatment for those patients in order to minimize potential toxic effects of the treatment and inconvenience associated with multiple clinic or hospital visits (7). Data from retrospective studies and prospective trials have shown that hypo fractionated radiation therapy delivering a slightly lower dose of radiation can achieve similar results in elderly patients or patients with poor performance status in comparison with the standard 6-7 week course of radiation therapy with or without chemotherapy. Such shortened radiotherapy schedules would be beneficial for patients with limited life expectancy (8). with advent of highly précised radiotherapy as three dimensional conformal radiotherapy( (3DCRT) and intensity modulated radiotherapy(IMRT) planning and delivery has the general goal of conforming the shape of a target volume, and limiting dose to critical normal structures while increasing the tumor dose. This study evaluates the efficacy and safety of a conformal hypo fractionation scheme biologically near conventional fractionation.

\section{Patients and Methods}

The current work is a prospective study that included thirty patients with pathologically confirmed high grade malignant glioma, carried at clinical oncology and nuclear medicine department, Zagazig University Hospitals between July2012 to January 2014 . The study protocol was approved by the local ethics committee at Zagazig University. The eligible patients were patients with newly diagnosed 
pathologically confirmed high grad gliomas, with karnofsky performance status (KPS) $\geq 50$, no previous cranial RT, o other active cancer, and age of patient $\geq 18$ years. Every patient in the study was subjected to history and physical examination including neurological examination, chemistry profile, brain MRI.

Treatment schedule: After surgery eligible patients started treatment by 3D conformal hypofracionation radiotherapy 45 Gy, 3 Gy per day, 5- days per week for 3 weeks for cases with gross total surgical resection and add boost dose of 9 Gy in three fractions to the area of the residual mass, with a $1-\mathrm{cm}$ safety margin, for a total dose of 54 Gy for cases with subtotal excision or biopsy alone.

\section{Radiation therapy technique:}

PTV was delineated on planning CT scan covering the contrast enhanced zone plus edema and $2 \mathrm{~cm}$ margin aiming at $45 \mathrm{~Gy}$ in 15 fractions for cases with gross total excision followed by 9 Gy in 3 fractions to the area of contrast enhanced zone plus $1 \mathrm{~cm}$ margin in cases with subtotal or biopsy. Fixation of the patient's head in treatment position was done using a thermoplastic mask after the patient was centralized. Radio-opaque markers were placed over the mask along laser beams to identify the reference isocenter. with scan slice thickness of $5 \mathrm{~mm}$. CT data were then transferred to the computer treatment planning system, where gross target volume (GTV), clinical target volume (CTV), planning target volume (PTV) and critical structures (two eyes and lenses, optic nerves \& chiasm, brain stem, spinal cord) were determined on each slice. Beam's eye view of PTV was used to choose beam directions and to shape fields with appropriate shielding. Dose distributions were then calculated. A 3D plan was done for each patient and was evaluated by isodose distribution in each slice. Dose volume histogram (DVH) was also done where the plan was considered acceptable if $95 \%$ of PTV was encompassed by $95 \%$ of prescribed tumor dose. Treatment was given isocentrically using a linear accelerator. The field entry points are aligned using laser beams according to reference points during setup process. All patients were treated by photon beams either $\mathrm{x}$ - rays produced by linear accelerator machine at 6 to $15 \mathrm{MV}$. Corticosteroid therapy (Dexamethasone ampoules) was given at a starting dose of $16 \mathrm{mg} /$ day divided dose and the dose was adjusted upward or downward to reach the minimum dose necessary to control neurological symptoms.

\section{Treatment evaluation and follow -up:}

The patients were monitored during treatment every week for possible acute radiation complications in the form transient worsening of symptoms due to edema and others as radiation dermatitis and alopecia that need useful management and assurance. After completion of RT, patients were evaluated monthly (or after clinical signs of a recurrence) by physical and neurologic examination, determination of performance status, and every 3 months by imaging with serial CT scan or MRI.

\section{Response criteria:}

Complete response (CR): complete disappearance of all detectable disease and reversion of all radiological examinations to normal, by two observations not less than four weeks apart.

Partial response (PR): $50 \%$ or more decrease in tumor area multiplication of longest diameter by the greater perpendicular diameter), determined by two observations not less than four weeks apart.

Stationary disease (SD): A 50\% decrease in total tumor size cannot be established, nor has a $25 \%$ increase in its size been demonstrated.

Progressive disease (PD): $25 \%$ or more increase in size of the lesion or appearance of new lesion.

\section{Statistical Analysis}

All data were collected, tabulated and statistically analyzed using SPSS 15.0 for windows. Continuous Quantitative variables e.g. age were expressed as the mean \pm SD \& median (range), and Categorical Qualitative variables were expressed as an absolute frequencies "number' and relative frequencies (percentage). Categorized data were compared using the Chi-square $\left(\chi^{2}\right)$ test, as count in some cell was less than 20 so we used Fisher's exact correction. Overall Survival (OAS) was calculated as the time from surgery to death or date last known alive (censored). Progression Free Survival (PFS) was calculated from time of achievement of a certain response to date of progression. Stratification of OS, and PFS was done according to all possible prognostic factors. These time-to-event distributions were estimated using the method of Kaplan-Meier, and compared using two-sided exact stratified log-rank tests. The Cox proportional hazards regression model was used to identify the independent prognostic factors as well as to estimate their effects on OS \& PFS All variables found significant on univariate Cox regression were considered for multivariate Cox regression $<$ 0.05 was considered statistically significant $(\mathrm{S}), \mathrm{P}<0.005$ was considered highly statistically significant (HS), and P > 0.05 was considered non statistically significant (NS).

\section{Results}

\section{Patients' characteristics}

This prospective study included thirty patients with brain glioma. Median age was 53years (range: 26-66 years). Twenty patients $(66.7 \%)$ were males and ten patients $(33.3 \%)$ were females with male to female ratio of $2: 1$. The majority of our patients had KPS of 50-70\% (19 patients; 63.3\%), and presented with manifestations of increased intracranial tension (headache: 24 patients, $80 \%$ and vomiting; 10 patients, 33\% and blurring of vision;20 patients, $66 \%$ ). 18 
patients (60\%) had GBM, and 19 patients $(63,3 \%)$ underwent subtotal resection, 9 patients $(30 \%)$ underwent biopsy and only 2 patients(6.7) had near complete excision.(Table 1).

Table 1. Characteristics of the patients.

\begin{tabular}{|c|c|c|}
\hline Variable: & No & $\%$ \\
\hline \multicolumn{3}{|c|}{${ }^{8}$ Age: } \\
\hline$<50$ & $\overline{14}$ & 46.7 \\
\hline$\geq 50$ & 16 & 53.3 \\
\hline \multicolumn{3}{|c|}{ sSex: } \\
\hline Male & 20 & 66.7 \\
\hline Femal & 10 & 33.3 \\
\hline \multicolumn{3}{|c|}{ spS: } \\
\hline$>70$ & 11 & 36.7 \\
\hline$\geq 50-70$ & 19 & 63.3 \\
\hline \multicolumn{3}{|c|}{ "Histologic grade: } \\
\hline Grade III & 12 & 40 \\
\hline Grade IV & 18 & 60 \\
\hline \multicolumn{3}{|c|}{ 'Type of surery: } \\
\hline Biopsy & 9 & 30 \\
\hline Subtotal resection & 19 & 63.3 \\
\hline Near total resection & 2 & 6.7 \\
\hline \multicolumn{3}{|c|}{ sclinical presentation: } \\
\hline Headache & 24 & 80 \\
\hline Vomiting & 10 & 33.3 \\
\hline Blurring of vision & 20 & 66.7 \\
\hline Seizures & 12 & 40 \\
\hline
\end{tabular}

\section{Radiologic response:}

Follow up radiologic evaluation after four to six weeks of radiotherapy showed that two patients $(6.7 \%)$ had complete response, twenty patients $(66.7 \%)$ had partial response, six patients $(20 \%)$ had stationary course and 2 patients $(6.7 \%)$ had progressive course.

\section{Survival analysis}

The median follow up was 12.23 months (range: 4-22 months). During follow-up period, 13 patients (43.3\%) died from their illness and 17 patients $(56.6 \%)$ were still alive at the last follow-up. Of these patients (2/7) maintained complete response, (5/7) maintained partial response, (13/23) showed progressive disease after partial response and (10/23) showed stable disease after partial response. One-year OAS and PFS rates were $58.9 \%$ and $12 \%$, respectively. Patients with $<50$ years of age showed one-year OAS and PFS of $85 \%$, and $25 \%$, respectively compared to $39 \%(\mathrm{p}=0.012$, $\mathrm{HR}=0.188$ and $95 \% \mathrm{CI}=0.051-0.694)$ and $0 \%(\mathrm{p}=0.007$, $\mathrm{HR}=0.301$ and $95 \% \mathrm{CI}=0.126-0.718$ ), for older patients(Figure 3 and 4). For patients with KPS of $>70 \%$, one-year OAS and PFS rates were $90 \%$ and respectively $20 \%$, compared to $39 \%(\mathrm{p}=0.020, \mathrm{HR}=0.88$ and $95 \% \mathrm{CI}=0.011-$ $0.681)$ and $7 \%(\mathrm{P}=0,031$, $\mathrm{HR}=0.371$ and $95 \% \mathrm{CI}=0.151-$ $0.913)$ ), respectively, for patients with KPS between $50 \%$ and $70 \%$ (Figure 2 and 5). For patients with grade III , One-year OAS and PFS rates were $100 \%$ and $0 \%$ respectively, compared to $29 \% \quad(\mathrm{p}=0.945$, HR- 0.00 , $95 \% \mathrm{CI}=0.073-1.002)$, and $25 \%((\mathrm{p}=0.002, \mathrm{HR}=0.203$, and $95 \% \mathrm{CI}=0.073-0.564)$, respectively, for those with grade IV(Figure 1 and 6). Patients who underwent near resection showed one-year OAS and PFS rates of $100 \%$ and $50 \%$, respectively, compared to $72 \%((\mathrm{p}=0.004, \mathrm{HR}=0.195$ and $95 \% \mathrm{CI}=0.064-0.596)$ ), and $12 \%(\mathrm{p}<0.001$, HR: $5.3,95 \%$ CI: 2.4-11.5), multivariate Cox proportional hazard ratio regression analysis for potential independent predictors for Overall Survival (OAS) in this study showed that age and KPS are the most prognostic factors predicting overall survival where age $\leq 50$ years has longer OS compared to age $>50$ years $(\mathrm{p}=0.010, \mathrm{HR}=0.148,95 \% \mathrm{CI}=0.034-0.641)$ and KPS $>70 \%$ has longer OS compared to $>50-70 \%$ (p $=0.015, \mathrm{HR}=0.078$ and $95 \% \mathrm{CI}=0.009-0.603)$. This overall model fit: Chi-square $=18.352$, d.f. $=2, p<0.001$ (HS)(Table 2). But Multivariate Cox proportional hazard ratio regression analysis for potential independent predictors for Progression Free Survival (PFS) showed that grade of the tumor and overall response were the most prognostic factors that predict progression free survival where grade III has longer PFS compared to grade IV( $p=0.002, H R=0.202$ and $95 \%$ $\mathrm{CI}=0.071-0.574)$ and overall response has longer PFS compared with $\mathrm{NR}(\mathrm{p}-0.014, \mathrm{HR}=0.211$ and $95 \% \mathrm{CI}=$ $0.061-0.726)$. This Overall model fit: Chi-square $=15.389$, d.f. $=2, p<0.001$ (HS)( Table 3) and Variable not included in the older Age $\leq 50 \mathrm{y}, \mathrm{PS}>70 \%$ and total resection.

The treatment schedule was well tolerated and there were insignificant toxicity in the form of minimal side effects to radiation therapy, in the form of a transient worsening of pretreatment symptoms most of them was bothersome headache, early in the course of irradiation which usually responds to corticosteroids and analgesic. 


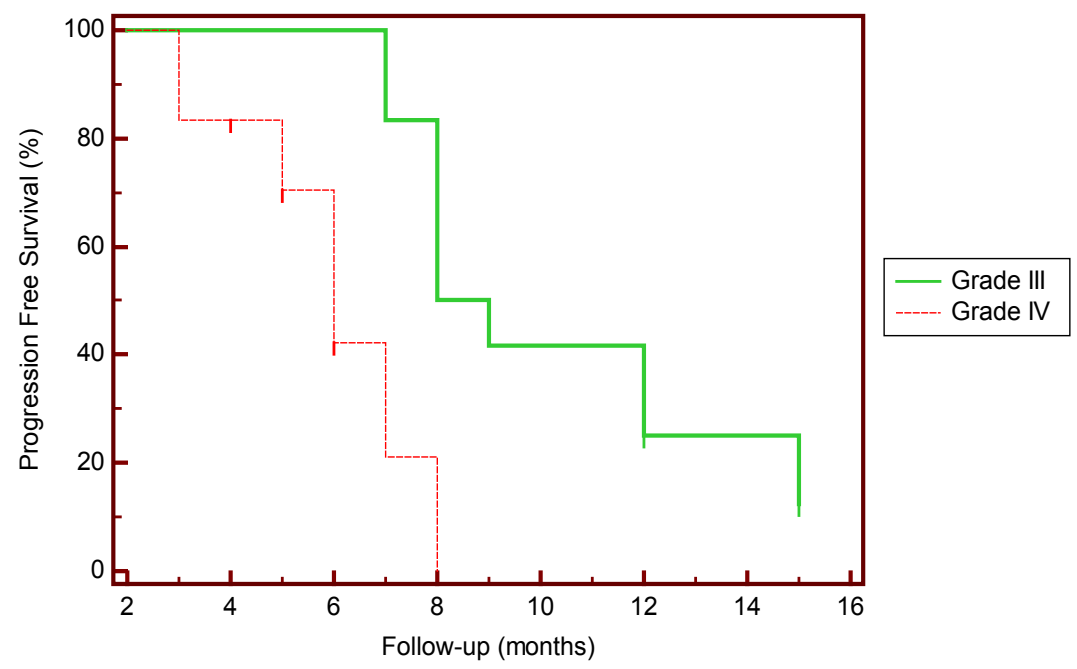

Number at risk

Group: Grade III

$\begin{array}{cccccccc}12 & 12 & 12 & 6 & 5 & 2 & 2 & 0 \\ \begin{array}{c}\text { Group: Grade IV } \\ 18\end{array} & 13 & 4 & 0 & 0 & 0 & 0 & 0\end{array}$

Figure 1. Kaplan-Meier estimates of Progression Free Survival (PFS) by histopathological grade

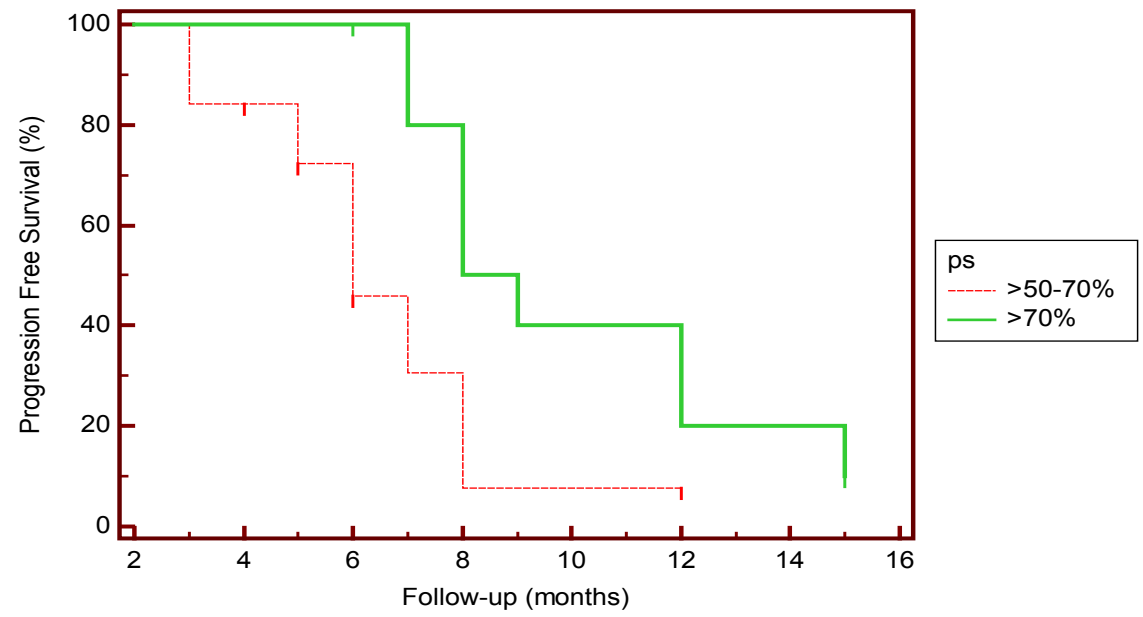

Number at risk

\begin{tabular}{|c|c|c|c|c|c|c|c|}
\hline Group: >50- & & & & & & & \\
\hline 19 & 14 & 6 & 1 & 1 & 0 & 0 & 0 \\
\hline Group: $>70^{\circ}$ & & & & & & & \\
\hline 11 & 11 & 10 & 5 & 4 & 2 & 2 & 0 \\
\hline
\end{tabular}

Figure 2. Kaplan-Meier estimates of Progression Free Survival (PFS) by performance status. 


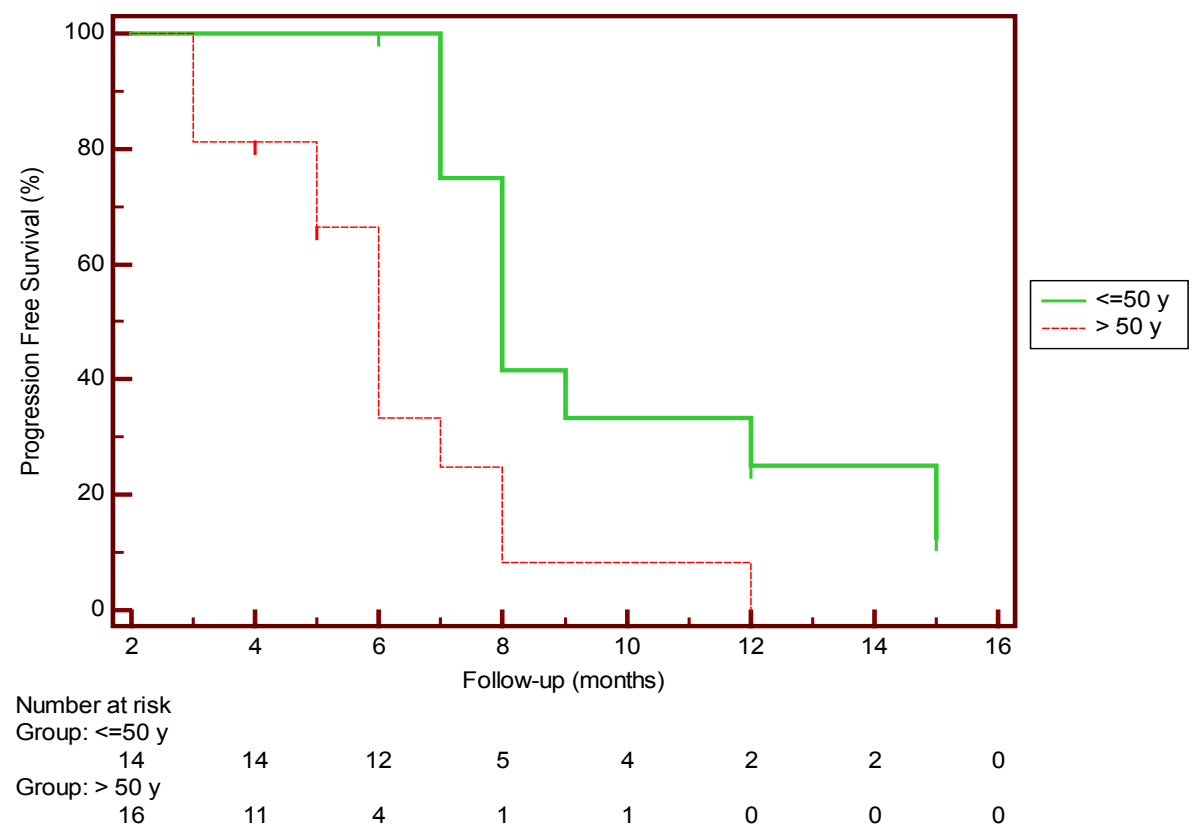

Figure 3. Kaplan-Meier estimates of Progression Free Survival (PFS) by age group,

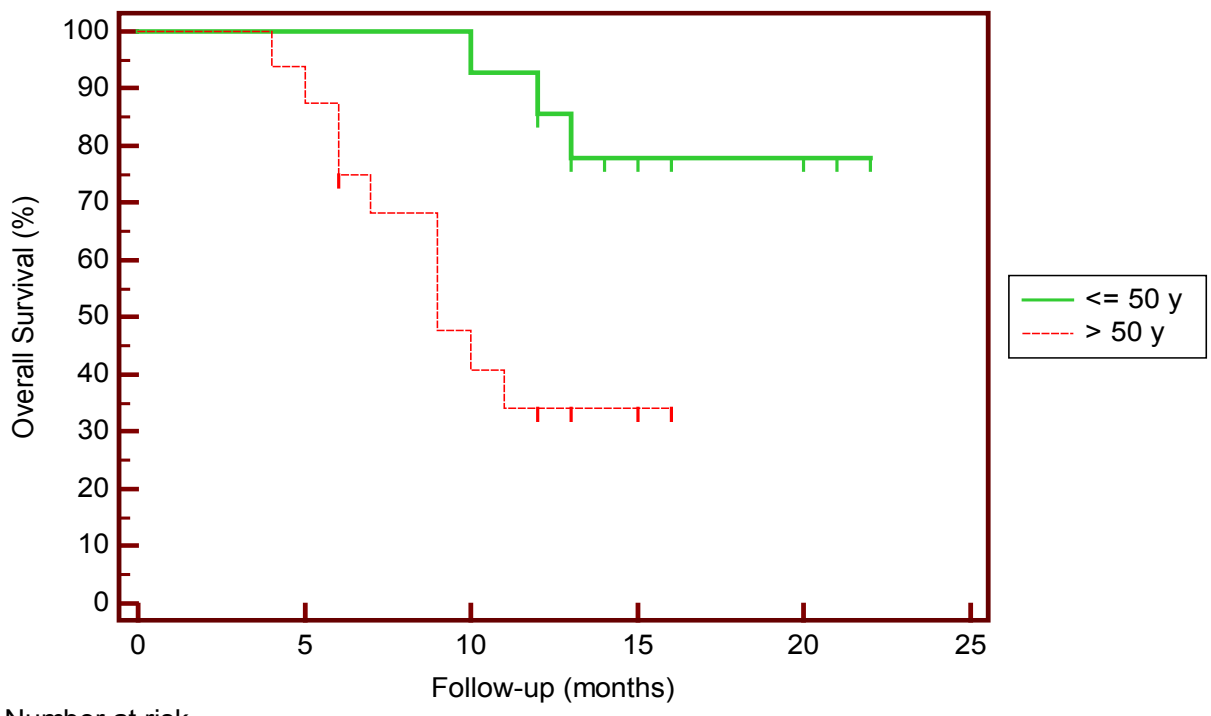

Number at risk

Group: $<=50$ y

Group: $>50$ y

14

13

5

3

0

16

14

6

1

0

0

Figure 4. Kaplan-Meier estimates of Overall Survival (OS) by age group 


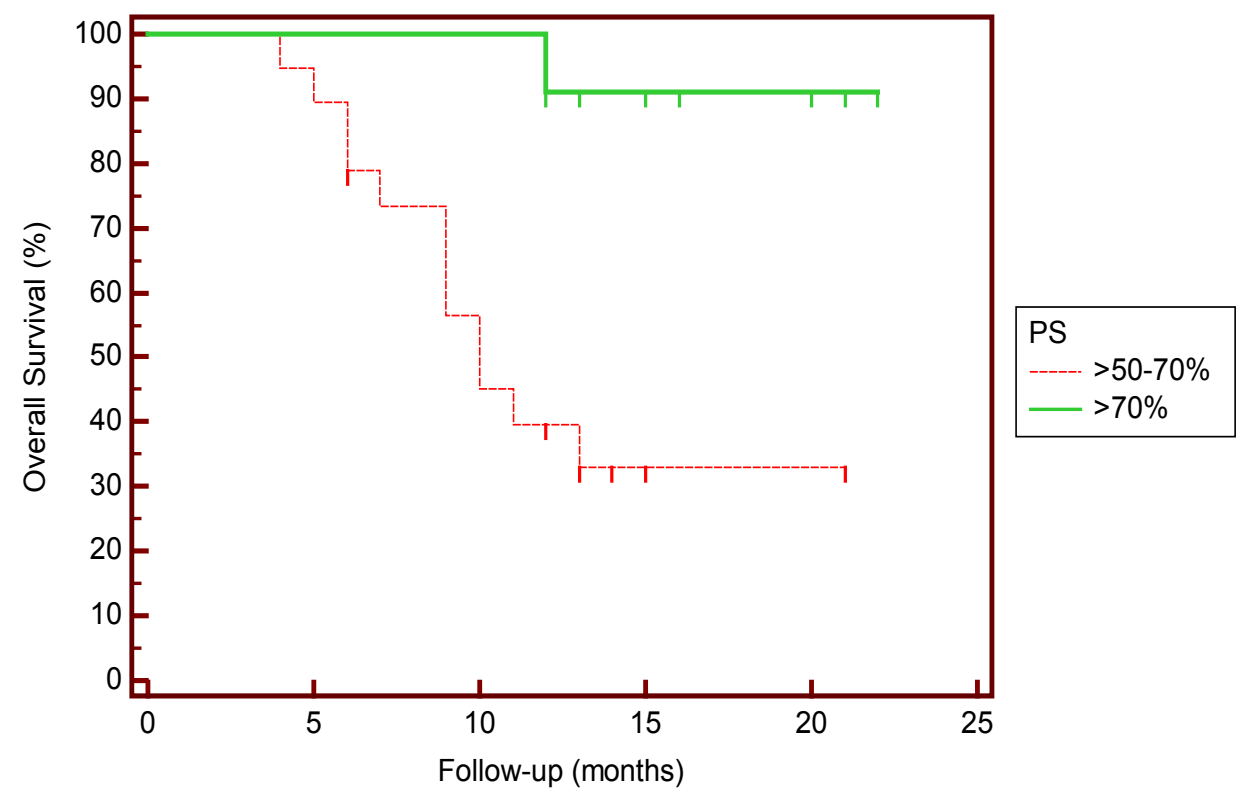

Number at risk

Group: $>50-70 \%$

$\begin{array}{cccccc}19 & 17 & 8 & 1 & 1 & 0 \\ \text { Group: }>70 \% & 11 & 11 & 5 & 2 & 0\end{array}$

Figure 5. Kaplan-Meier estimates of Overall Survival (OS) by performance status.

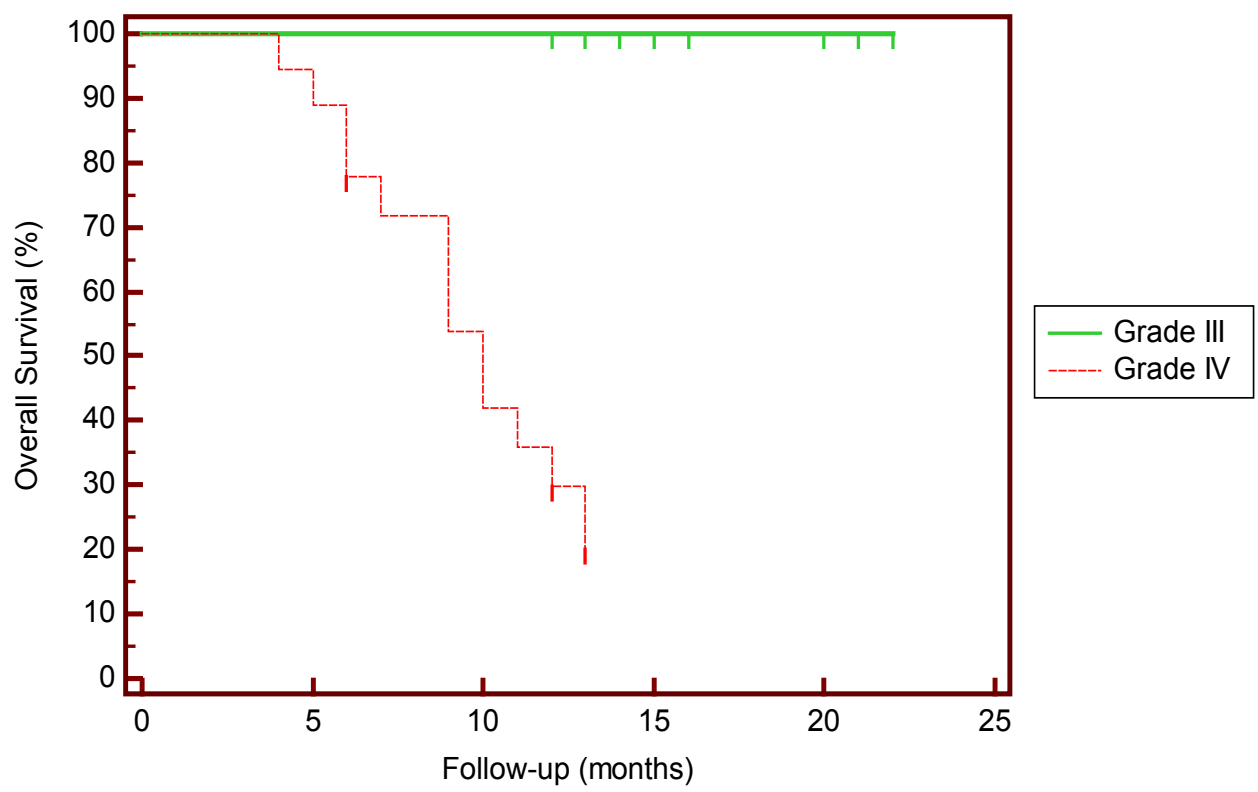

Number at risk

Group: Grade III

$\begin{array}{cccccc}12 & 12 & 12 & 6 & 3 & 0 \\ \text { Grade IV } & & 7 & 0 & \end{array}$

18

7

0

0

Figure 6. Kaplan-Meier estimates of Overall Survival (OS) by histologic grade. 
Table 2. Univariate Cox proportional hazard ratio regression analysis for potential independent predictors for Progression Free Survival (PFS)and overall survival(OAS)

\begin{tabular}{|c|c|c|c|c|c|c|}
\hline Factor & medianPFS & $\begin{array}{c}\text { one year PFS } \\
\text { (months) }\end{array}$ & $\begin{array}{l}\text { P value } \\
(\%)\end{array}$ & median (OS) & $\begin{array}{l}\text { one year(OS) } \\
\text { (months) }\end{array}$ & $\begin{array}{l}\text { P value } \\
(\%)\end{array}$ \\
\hline *Age(no) & & & P-0.007 & & & $\mathrm{P}=0.02$ \\
\hline$<50(14)$ & 8 & 25 & $\mathrm{HR}=0.301$ & NR & 85 & $\mathrm{HR}=0.188$ \\
\hline$\geq 50(16)$ & 6 & 0 & $95 \% \mathrm{CI}(0.126-0.718)$ & 9 & 34 & $95 \%$ CI (0.051-0.694) \\
\hline * Sex(no) & & & $\mathrm{P}=0.463$ & & & $\mathrm{P}=0.381$ \\
\hline Male(20) & 8 & 12 & $\mathrm{HR}=1.406$ & NR & 60 & $\mathrm{HR}=0.650$ \\
\hline Female(10) & 7 & 13 & $95 \% \mathrm{CI}(0.567-3.485)$ & 13 & 57 & $95 \% \mathrm{CI}(0.540-5.042)$ \\
\hline *PS & & & $\mathrm{P}=0,031$ & & & $\mathrm{P}=0.020$ \\
\hline$>70 \quad(11)$ & 6 & 20 & $\mathrm{HR}=0.371$ & NR & 90 & $\mathrm{HR}=0.088$ \\
\hline$\geq 50-70(19)$ & 8.5 & 7 & $95 \% \mathrm{CI}(0.151-0.913)$ & 10 & 39 & $95 \% \mathrm{CI}(0.011-0.681)$ \\
\hline *Histologic grade: & & & $\mathrm{P}=0.002$ & & & $\mathrm{P}=0.945$ \\
\hline Grade III(12) & 8.5 & 25 & $\mathrm{HR}=0.203$ & NR & 100 & $\mathrm{HR}=0.000$ \\
\hline Grade IV(18) & 6 & 0 & $95 \% \mathrm{CI}(0.073-0.564)$ & 10 & 29 & $95 \% \mathrm{CI}(0.073-1.002)$ \\
\hline *Response: & & & $\mathrm{P}=0.008$ & & & $\mathrm{P}=0.013$ \\
\hline OAR(23) & 8 & 15 & $\mathrm{HR}=0.208$ & NR & 68 & $\mathrm{HR}=0.229$ \\
\hline NO response(7) & 6 & 0 & $95 \% \mathrm{CI}(0.065-0.664)$ & 7 & 28 & $95 \% \mathrm{CI}(0.072-0.730)$ \\
\hline *Type of surgery: & & & $\mathrm{P}=0.163$ & & & $\mathrm{P}=0.004$ \\
\hline Biopsy(9) & 6 & 0 & $\mathrm{HR}=0.303$ & 7 & 22 & $\mathrm{HR}=0.159$ \\
\hline $\operatorname{STR}(19)$ & 8 & 12 & $95 \% \mathrm{CI}(0.057-1.608)$ & NR & 72 & 95\% CI (0.073-I.002) \\
\hline NTR(2) & 11 & 50 & & NR & 100 & \\
\hline
\end{tabular}

NR (not reached), NTR(near total resection), OAL(overall response), PS(performance status), STR(subtotal resection,

\section{Discussion}

Although conventional radiotherapy with or without chemotherapy still the standard of care for patients with high grade glioma, Hypo fractionation has three advantages over standard treatment. First, the total treatment time is shorter. Second, the reduced number of treatment sessions has a cost advantage over standard treatment. Finally, a radio biologic advantage of hypo fractionation may existt. Conventional radiotherapy schedules (60-66Gy in 30-33 fractions) require 6-7 weeks affect the psycho-social state of the patient, reduce the quality of life and increase the coast.

Different hypo fractionated RT schedules for the treatment of high grade glioma has been described. A study conducted by Roa and colleagues), using hypo fractionated radiotherapy (40 Gy in 15 fractions over 3weeks) found similar survival of approximately 6 months with those received standard RT(9). French study found similar survival rates of 29 weeks on 39 patients treated by 50 Gy in 20 fractions over 4 weeks compared to 16.6 weeks in patients treated with best supportive care(10).Another study conducted by Piccirilli et al., reported a median survival 13.6 months on treating 11 elderly patients with temozolomide and radiotherapy (11). Aboziada and Abokresha treated patients with high grade glioma by hypo fractionated conformal irradiation (49.5 Gy/15 fractions/3 weeks). Two-year OAS and PFS rates were $68 \%$ and $60 \%$, respectively (12). Monjazeb and colleagues reported MS was 13.6 months, with 1 and 2 year survival rates of $57 \%$ and $19 \%$, respectively for good risk patients using IMRT hypo fractionation schedule (13). Other studies reported similar survival benefits in elderly patients treated with 30 Gy in 6 fractions over 2 weeks $(14,15$, and 16).

HFRT was initially developed as a pragmatic concept in poor-risk patients, but more recent studies have explored HFRT as a novel approach for achieving intratumoral dose escalation.

The current study included thirty patients with established diagnosis of high grade glioma following surgery were subjected to treatment with hypo fractionated radiotherapy $45 \mathrm{GY}$ in 15 fractions for cases with complete excision followed by $9 \mathrm{GY}$ in 3 fractions for cases underwent subtotal excision or biopsy. Our results compare favorably with other studies used hypo fractionated radiotherapy and other studies used chemo radiotherapy. These results suggests that hypo fractionation radiotherapy has comparable outcome to conventional fractionation. In our study the median progression free survival was eight months where sex month PFS represent 67.7 and twelfth months PFS represents $12.7 \%$ but fifteen months PFS account about 6.3\%.These results are comparable with others who reported progression free survival rates ranging from $6-8$ months $(\mathbf{1 7}, \mathbf{1 8}$, and 19).

In our study six months OAS rate represent $86.7 \%$ and eighteen months OS rate account for $54.7 \%$ but 1 year OS represent $58.8 \%$ of all cases These results are comparable with others who found one and two year survival rates of $57 \%$ and $19 \%$, respectively (13) and 6 and 12 months OS rates were $86 \%$ and $36 \%$ respectively $(\mathbf{2 0})$.

Multivariate Cox proportional hazard ratio regression analysis for potential independent predictors for Progression Free Survival (PFS) and overall survival(OS)(Table3) 
showed that grade of the tumor and overall response were the most prognostic factors that predict progression free survival where grade III has longer PFS compared to grade IV $(\mathrm{p}=$ $0.002, \mathrm{HR}=0.202$ and $95 \% \mathrm{CI}=0.071-0.574)$ and overall response has longer PFS compared with $\mathrm{NR}(\mathrm{p}=0.014, \mathrm{HR}=$ 0.211 and $95 \% \mathrm{CI}=0.061-0.726$ ). and showed that age and KPS are the most prognostic factors predicting overall survival where age $\leq 50$ years has longer OS compared to age $>50$ years $(\mathrm{p}=0.010, \mathrm{HR}=0.148,95 \% \mathrm{CI}=0.034-0.641)$ and KPS $>70 \%$ has longer OS compared to $>50-70 \%(\mathrm{p}$ $=0.015, \mathrm{HR}=0.078$ and $95 \% \mathrm{CI}=0.009-0.603)$. Abbreviated course of RT well tolerated with minimal radiation toxicity and associated with similar survival as for standard RT but with reduced toxicity $(\mathbf{1 2 , 2 0})$.

Table 3. Multivariate Cox proportional hazard ratio regression analysis for potential independent predictors for progression free survival (PFS) Overall Survival (OAS)

\begin{tabular}{|llcc|}
\hline \multicolumn{1}{|c}{ HR } & \multicolumn{2}{c|}{$95 \%$ CI for HR } & P-value \\
\hline & *Factors affecting PFS: & \\
Grade III & 0.202 & $0.071-0.474$ & 0.002 \\
OAR & 0.211 & $0.061-0.726$ & 0.014 \\
& *Factors affecting OS: & \\
Age $<50$ & 0.148 & $0.034-0.641$ & 0.010 \\
PS $>70$ & 0.073 & $0.009-0.603$ & 0.015 \\
\hline
\end{tabular}

\section{Conclusions}

Hypofractionated radiotherapy was effective and well tolerated as the conventional radiotherapy for patients with high grade gliomas. This nonconventional regimen may be considered as a radiotherapy regimen suitable for those patients with expected short term overall survival. With further studies this regimen may be the regimen of choice clinical practice especially if combined with chemotherapy.

\section{REFERENCES}

[1] Meral Y. Sayren, Bektas K, Bekir H et al. The results of hypofractionated radiotherapy in 31 patients with high-grade gliomas. Med Oncol; 2007, 24:379-383.

[2] Mizuhiko T - Tomoko E - Shinji N et al. A pilot study of hypo fractionated radiation therapy with temozolomide for adults with glioblastoma multiforme. J Neurooncol; 2011; 102:247-253.

[3] Simpson JR, Horton J, Scott C et al. Influence of location and extent of surgical resection on survival of patients with glioblastoma multiforme: results of three consecutive Radiation Therapy Oncology Group (RTOG) clinical trials. Int J Radiat Oncol Biol Phys; 1993; 26:239-244.

[4] Floyd NS, Shiao Y, Bin S et al. Hypofractionated intensity-modulated radiotherapy for primary glioblastoma multiforme. Int J Radiat Oncol Biol Phys; 2004; 58:7217265 .

[5] Halperin C. Malignant gliomas in older adults with poor prognostic signs. Getting nowhere, and taking a long time to do it. Oncology; 1995; 9:229-234.

[6] Chang EL, Yi W, Allen PK et al. Hypofractionated radiotherapy for elderly or younger with low-performance status glioblastoma patients: outcome and prognostic factors. Int J Radiat Oncol Biol Phys; 2003; 56:519-528.

[7] Sultanem K, Patrocinio H ,Lambert C et al. The use of hypofractionated intensity-modulated irradiation in the treatment of glioblastoma multiforme: preliminary results of a prospective trial. Int J Radiat Oncol Biol Phys; 2004; 58:247252.

[8] Simon S. Lo, and Eric L. Chang,: Hypofractionated Radiation Therapy for Glioblastoma. American Society of Clinical Oncology. 2011; 1092-9118/10/1-10.

[9] RoaW., Brasher P.M.A., Bauman G., et al: Ab-breviated course of radiation therapy in older patients with glioblastoma multiforme: A prospective randomized clinical trial. J. Clin. Oncol; 2004;22: 1593-1598.

[10] Keime G F., Chinot O., Taillandie L., et al.: Radiotherapy for glioblastoma in the elderly. N. Engl. J. Med; 2007; 356: $1527-1535$

[11] Piccirlli M., Landi A., Salvati M., et al.: Gliom-atosis cerebri treatment in 11 elderly patients. J. Exp. Clin. Cancer Res; 2006; 25: 183-187.

[12] Aboziada M A, Abo-Kresha A E: Hypofractionated conformal irradiation of patients with malignant glioma. Journal of the Egyptian National Cancer Institute); 2012; 24, 139-143.

[13] Monjazeb AM, Ayala D, Jensen C et al. A Phase I dose escalation study of hypofractionated IMRT field-in-field boost for newly diagnosed glioblastoma multiforme. Int $\mathrm{J}$ Radiat Oncol Biol Phys; 2012. 82:743-8.

[14] Bauman G., FisherI B., Halpren C., et al.: A prospective study of short course RT in poor prognosis glioblastoma multiforme. Int. J. Radiat Oncol Biol Phys; 1994; 29: 835-839.

[15] Thomas R., James N., Guerrero D., et al.: Hypo fractionated radiotherapy as palliative treatment in poor prognosis patients with high grade glioma. Radiother Oncol; 1994; 33: 113.

[16] Mcalees.J., Stenning S, Ashley S., et al.: Hypofractionated radiotherapy for poor prognosis malig-nant glioma: Matched pair survival analysis with MRC controls. Radiother Oncol; 2003; 67: 177-182.

[17] Jermic B., Shibamoto Y., Grujicic D., et al.: Short-course radiotherapy in elderly and frail patientswith glioblastoma multiforme. A phase II study. J. Neu-rooncol; 1999;44 (suppl. 1): $85-90$

[18] Lutterbach J, Ostertag C. What is the appropriate radiotherapy protocol for older patients with newly diagnosed glioblastoma? J Clin Oncol.; 2005; 23:2869-2870.

[19] Arslan M, Karadeniz AN, Aksu G et al. Postoperative hypofractionated radiotherapy in glioblastoma multiforme. J BUON; 2006; 11:39-42.

[20] Toson EA. Sharaf eldeen D A; el-zafarany M E., and Kasem M: Conformal Hypofractionated Radiotherapy Concomitant with and Followed by Temozolomide in High Grade Glioma in Elderly Patients. Med. J. Cairo Univ; 2012; 80, 1,799-805 